\title{
BATA-UNET: DEEP LEARNING MODEL FOR LIVER SEGMENTATION
}

\author{
Fatima Abdalbagi ${ }^{1}$, Serestina Viriri ${ }^{2}$ and Mohammed Tajalsir Mohammed ${ }^{3}$ \\ ${ }^{1}$ Department of Computer Science, Sudan University of Science and Technology, \\ Khartoum, Sudan \\ ${ }^{2}$ Computer Science Discipline, University of KwaZulu-Natal, Durban, South Africa \\ ${ }^{3}$ Department of Computer Science, Sudan University of Science and Technology, \\ Khartoum, Sudan
}

\begin{abstract}
In computer vision, image segmentation is defined as process of a partition of an image in a number of regions with homogeneous features. The region of our interest here is the liver. Prior to the deep learning revolution traditional handcrafted features were used for liver segmentation but with deep learning the features are obtained automatically. There are many semiautomatic and fully automatic approaches have been proposed to improve the liver segmentation procedure some of them use deep learning techniques for Segmentation and other one use a Classical Based method for Segmentation. In this paper we aim to enhance our previous work which we were proposed a Batch Normalization After All - Convolutional Neural Network (BATA-Convnet) model to segment the liver, where the Dice is equal to $0.91 \%$ when implement our BATA Convnet using MICCA dataset and Dice is equal to 0.84\% when implement it using $3 D$-IRCAD dataset. Here in this paper we propose BATA-Unet model for liver segmentation, it's based on Unet architecture as backbone but differ in we added a batch-normalization layer an after each convolution layer in both construction path and expanding path. The proposed method was able to achieve highest dice similarity coefficient than the previous work where for MICCA dataset Dice $=0.97 \%$ and for $3 D$-IRCAD dataset $=0.96 \%$. Also our proposed model outperformed other state-of-the-art model when we compare it with them.
\end{abstract}

\section{KEYWORDS}

Liver segmentation, Deep learning, Computed Tomography, Handcrafted, U-NET

\section{INTRODUCTION}

Medical images are considering as a set of techniques, processes of creating visual representations (images) inside a body for clinical analysis and medical intervention. Medical imaging is today an invaluable tool for diagnosis and treatment planning. Imaging modalities such as X-ray, Ultrasound (US), Single photon emission computed tomography (SPECT), Positron emission tomography (PET), computed tomography (CT) and magnetic resonance imaging (MRI). The increasing introduction of digital imaging modalities allows efficient storage thereof and what is more important, the possibility of image processing and analysis to obtain quantitative data from them [1].

Liver segmentation process is aim to divide the pixels of the image depending on certain criteria into two groups: pixels that belong to the object of interest (liver) and the rest of pixels that doesn't belong to the liver. It's essential tasks in oncological therapy monitoring and radio therapeutic treatment where tumor information is vital for correct dosimetry calculations [2]. 
Signal \& Image Processing: An International Journal (SIPIJ) Vol.11, No.5, October 2020

Typically, this has been manually done by trained clinicians but it is time consuming and requiring much effort and also it subjective because of observer variability; as the result of that, an automatic liver segmentation system would thus be a great boon for perform these tasks. Because of complexity of liver shapes and variable liver sizes among patients the segmentation of the liver from medical images is very difficult and also due to low contrast between the liver and surrounding organs like stomach, pancreas, kidney and muscles [3].

There are many approaches have been proposed for liver segmentation procedure some of them use deep learning techniques for Segmentation and other one use a Classical Based method for Segmentation such as: region growing, clustering, deformable models or level sets, statistical shape models (SSMs), probabilistic atlases, graph cuts and recently, deep convolution neural networks [4][5].

Deep learning field is a new subfield of machine learning admits to learning representations from data that puts an emphasis on learning successive layers of increasingly meaningful representations. There is no long need to the term of "just like our minds" which hypothetical links between deep learning and biology. Deep learning is a mathematical framework for learning representations from data[6]. Recently, deep learning has achieved state-of-the-art performance in medical image analysis [7]. Deep learning techniques have been applied to medical image analysis with the intention of letting computers learn the features that optimally represent the problem data at hand [8]. The main applications that deep learning techniques are used for are classification, detection and segmentation [9].

The organ of interest in this research work is the liver. We focus on segmenting the liver from abdominal CT scans because it plays an important role in the study of the liver functions and can assist in the diagnosis of liver diseases. Accurately detecting and delineating the liver separates the liver from its surrounding organs and isolates it for complicated analysis (Gotra et al., 2017). Segmenting the liver may reduce the computation time required in analysis as the liver only occupies a portion of the abdominal CT scan. Accurate liver segmentation ensures that the whole liver is analysed and the surrounding organs and tissues are eliminated. Inspired by the use of deep learning in particular Unet and its success in organ segmentation, an automatic liver segmentation method using Unet architecture is proposed in this research work.

U-NET is an artificial neural network kind of Convolutional Neural Networks (ConvNets) approach that is able to produce visual information. When visualize U-Net architecture it appears similar to the letter $U$ so it takes its name from here. U-Nets was first proposed by Olaf Ronneberger, Phillip Fischer, and Thomas Brox in 2015 for Bio Medical Image Segmentation and in 2016 within Christ et al work for automatic liver segmentation on CT Scan images. The great idea about U-Net is that it is able to receive an image as input and produce another image as output with size equal to the size of input, which is pretty useful for generating segmentation images.

The rest of this paper is organized as follows: in section 2 the literature review and related work are provided and section 3 briefly describes the methods and techniques used in this work. The results are discussed in section 4. Finally, the conclusions are drawn in section 6.

\section{RELATED WORK}

There are many methods which perform liver segmentation using different criteria for grouping the voxel, most of these methods are based on image-based and prior model-based; where each of 
them has some limitations. For example the image-based methods have a drawback in that they need user interaction, more refinement and may be sensitive to initial contours [10]

In [10], a fully automatic framework was proposed for liver segmentation based on 3D convolutional neural network (CNN) and globally optimized surface evolution. Firstly, the deep 3D CNN gives the initial liver surface after it was trained to learn a subject-specific probability map of the liver. Then, refining the initial liver segmentation by using the prior information about novel energy function; Finally, propagated the initial liver surface to the optimal position by minimized the energy function using a global optimization-based approach.

[11] They develop a fully automatic liver segmentation framework without any user interaction. Which combine a deep learning algorithm and graph cut approach; mainly their framework consists of two main steps: (1) liver detection and segmentation using convolutional neural networks(CNN) model which used to learn the liver likelihood map to automatically identify the liver surface. (2) Refinement the initial liver segmentation from the first step by incorporating the learned liver probability map into a graph cut model. To evaluate their framework, they use 40 contrast enhanced CT volumes from two public databases MICCAISliver07 and 3Dircadb. They found that their framework can increase the efficiency of the physician.

[12] present a combined automatic segmentation of the liver and its lesions in CT and MRI abdomen images using two cascaded fully convolutional neural networks (CFCNs) one for the segmentation of the liver and the other for its lesions. They use an abdominal CT dataset comprising 100 hepatic tumor volumes for training the CFCN models. In the first step of their work they pre-processed the CT/MRI image with HU-windowing or N4 bias correction. Then in second step, segmenting the liver from abdomen CT/MRI scans using first pertained FCN after that they use the output of last step to be input for a second FCN which segments lesions from the given segmented liver ROI. And finally last step was the post-processing using 3D Conditional Random Field (3D CRF). Their results show that the CFCN achieves Dice scores over 94\% for the liver with computation times below 100s per volume. And in contrast to prior work their method could be generalized to segment multiple organs in medical data using multiple cascaded FCNs.

Ben-Cohen et al proposed a fully convolutional network (FCN) network architecture for liver segmentation and liver metastases detection in CT examinations [13]. They trained two networks, one for liver segmentation and the other for lesion detection. Two framework variations were used for evaluation as well as the initial framework. The segmentation performance was evaluated using the Dice index, sensitivity and positive predicate values (PPV). The FCN-8s with 3 slices produced a Dice of 0.89 , the FCN-8s produced a Dice of 0.88 and the FCN- $364 \mathrm{~s}$ with 3 slices produced a Dice of 0.87 . The FCN-8s with 3 slices produced the best results with an average sensitivity of 0.86 and an average PPV of 0.95 . The lesion detection performance was evaluated using two metrics: true positive rate (TPR) and false positive per case (FPC). The dataset used is the MICCAI-SLiver07 challenge dataset. The FCN-4s with 3 slices performed the best producing a TPR of 0.88 and a FPC of 0.74 .

This study [14] introduced a CNN for liver segmentation on abdominal computed tomography (CT) images with focusing on the performance of generalization. They show high generalization performance and accuracy. They proposed an auto-context neural network; it achieved an effective estimation to obtain the shape prior. They use a self-supervised contour scheme to extend their network. They achieved a better accuracy when compared to the state-of-the-art networks by reducing $10.31 \%$ of the Hausdorff distance. 
Signal \& Image Processing: An International Journal (SIPIJ) Vol.11, No.5, October 2020

In this paper [15], the authors propose some modifications to U-Net model architecture by developing a novel model called MultiResUNet which is an enhanced version of U-Net. They hypothesis that there was a contradiction between the features passed from the encoder network and the features propagating through the decoder network and to harmonize this contradiction they proposed additional processing called Respaths, also to increase the ability of multiresolution analysis they proposed MultiRes blocks which was inspirations from Inception blocks. They test their model using five variety medical image datasets of different modalities. The five datasets are Murphy Lab, ISBI-2012, ISIC-2018, CVC-ClinicDB and BraTS17. They had obtained a relative improvement in performance of $10.15 \%, 5.07 \%, 2.63 \%, 1.41 \%$, and $0.62 \%$ respectively. They also experimented with a 3D version of MultiResUNet, and it outperforms the standard 3D U-Net as well.

A literature review of medical image segmentation based on U-net was presented by [16]. They were focused on the successful segmentation experience of U-net in six medical imaging systems including computed tomography (CT), magnetic resonance imaging (MRI), ultrasound, X-ray, optical coherence tomography (OCT), and positron emission computed tomography (PET). There are many kinds of lesion regions extracted by these application. Also in this study, they were introduced the method of combining the original U-net architecture with deep learning and a method for improving the U-net network. They came out that the six imaging systems mentioned in their article, are not perfect in some imaging systems. Consequently, it needs to be improved in future studies for application in various imaging systems

A new, more powerful deeply-supervised encoder-decoder segmentation architecture based on nested and dense skip connections named U-Net++ was proposed by [17]. U-Net++ is differ from U-Net in that the feature maps of the encoder are directly received in the decoder in U-Net but in U-Net++ model makes them undergo a nested convolution block. They evaluated their proposed architecture in comparison with U-Net and wide U-Net architectures across four medical imaging datasets include lung nodule segmentation, colon polyp segmentation, cell nuclei segmentation, and liver segmentation. They came out that U-Net++ with deep supervision achieved an average IoU gain of 3.9 for U-Net and 3.4 points for wide U-Net. According to results of their experiments, the proposed architecture in this study was effective, yielding significant performance gain over U-Net and wide U-Net. U-Net++ was used in comparative in this study [18] where it was compared with two other deep learning models :CE-Net and MultiResUNet for the segmentation of Corpus Callosum in the Brain MRI images.

Two deep encoder-decoder convolutional neural networks (EDCNN) were constructed and trained to cascade segments of both the liver and lesions in CT images in this paper [19].the first EDCNN was responsible for segmented the liver image which was been an input for the training of a second EDCNN; where it then responsible for segments the tumor regions within the liver ROI regions as predicted by the first EDCNN. In order to evaluate their performance, the proposed EDCNN networks produced an average DICE score of $95.22 \%$ for the test set of CT images using a public dataset (3DIRCADb) and it was compared with some of the existing methods. The experimental results demonstrated that the proposed EDCNN achieved improved performance in segmentation accuracy over some existing methods.

Modified U-Net (mU-Net) network architecture was proposed by [20],they was depend on processing an object-dependent up sampling and restructures the residual path and the skip connection by combines features in the residual path into features in the skip connection. the mUNet is differ from the U-Net architecture it handles edge information and morphologic information of the objects more effectively than the U-Net. Furthermore, in order to extract high level global features of small object inputs the proposed architecture has additional convolution 
Signal \& Image Processing: An International Journal (SIPIJ) Vol.11, No.5, October 2020

layers in the skip connection. The proposed modified U-Net (mU-Net) was evaluated using two public datasets which are Liver tumor segmentation (LiTS) challenge 2017 and 3D Image Reconstruction for Comparison of Algorithm Database (3Dircadb). They came out of this result for liver segmentation respectively: DSC of $98.51 \%$, DSCs was $96.01 \%$ for the liver segmentation.

\section{MeThODS AND TECHNIQUES}

In this paper, a BATA-Unet model for liver segmentation based on U-Net architecture is proposed. The main advantage of using Unet architecture comes from ability of U-Net to predict a good segmentation map by combining the localisation information from the construction path with the contextual information in the expanding path which result of obtaining a general information. Another advantage is that the input images of different sizes can be used for training the network because there is no need for dense layer.

Our Methodology for Liver segmentation is shown in the flowchart in Figure.1. It consists of four steps: First is pre-processing the CT images. Second step is training the BATA-Unet model for liver segmentation. Then, testing the trained network to produces a probability map as a subject specific prior, which assigns each pixel the likelihood of being the liver for the target image. Last step is post-processing step to maximize the result efficiency.

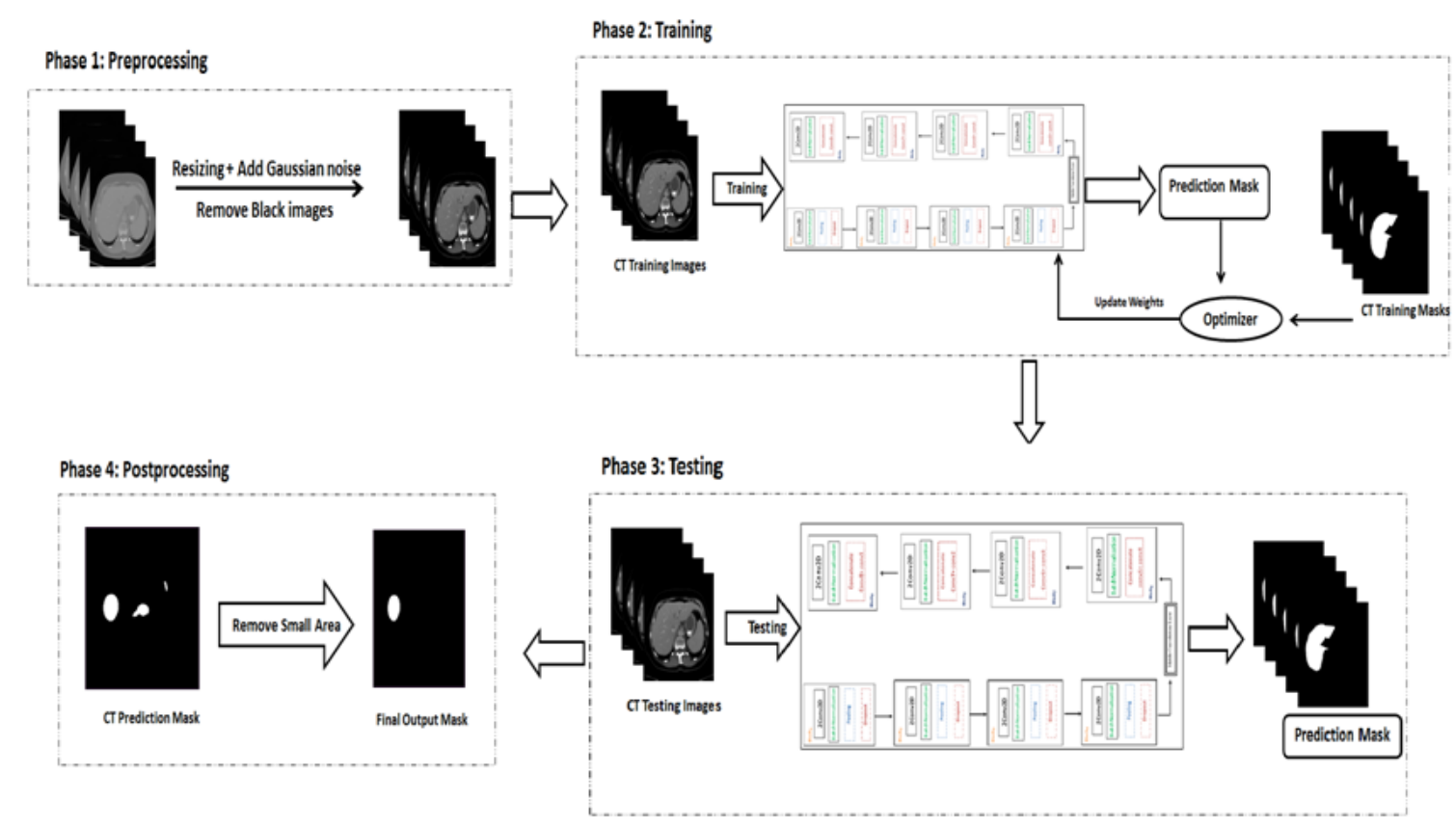

Figure.1 The flowchart of our proposed BATA-U-Net model.

\subsection{Datasets}

Generally, gathering and organizing of the medical image dataset is one of the most exhausting and hard jobs especially for the researchers as well as for the physicians. Producing of such a data which can be effectively used in computer vision for the segmentation problem comes as one of the major challenges due to various reasons such as small number of input data and the issues of the patient information privacy. The datasets that has been used in this work are Medical Image Computing and Computer Assisted Intervention (MICCAI) dataset and 3D-Image Reconstruction 
Signal \& Image Processing: An International Journal (SIPIJ) Vol.11, No.5, October 2020

for Comparison of Algorithm Database (3D-IRCAD). The MICCAI images are enhanced with different operator and the pixel disbanding varied somewhere in the range of 0.55 and $0.80 \mathrm{~mm}$ and inter-slice distance varied from 1 to $3 \mathrm{~mm}$. most of the image includes tumors, metastasis and sores of different sizes. It comprises of 20 volume images with corresponding ground truth segmentations used for training and for testing it have 10 volume images. All CT image in 3DIRCAD database are in DICOM and VTK format of size $512 \times 512$ pixels. It is composed of 20 patients 10 men and 10 women with hepatic tumours in $75 \%$ of cases with handmade true segmentation for liver, bones, tumors and others by medical specialists. The size of these images is $512 \times 512$, but may take a very long time to complete training such as images on personal computer, so the images were scaled down to $128 \times 128$ pixels.

\subsection{U-Net Architecture}

The U-NET architecture is shown in Figure.2, it contains two paths: contraction path (also called as the encoder) and expanding path (also called as the decoder). The encoder part is used to capture the context in the image using convolutions layer. The decoder part is used to enable precise localization using transposed convolutions[21]. The main idea behind the U-Net is that during the training phase the first half which is the contracting path is responsible for produce the relevant information by minimizing a cost function related to the operation desired and at the second half which is the expanding path the network it would be able to construct the output image.

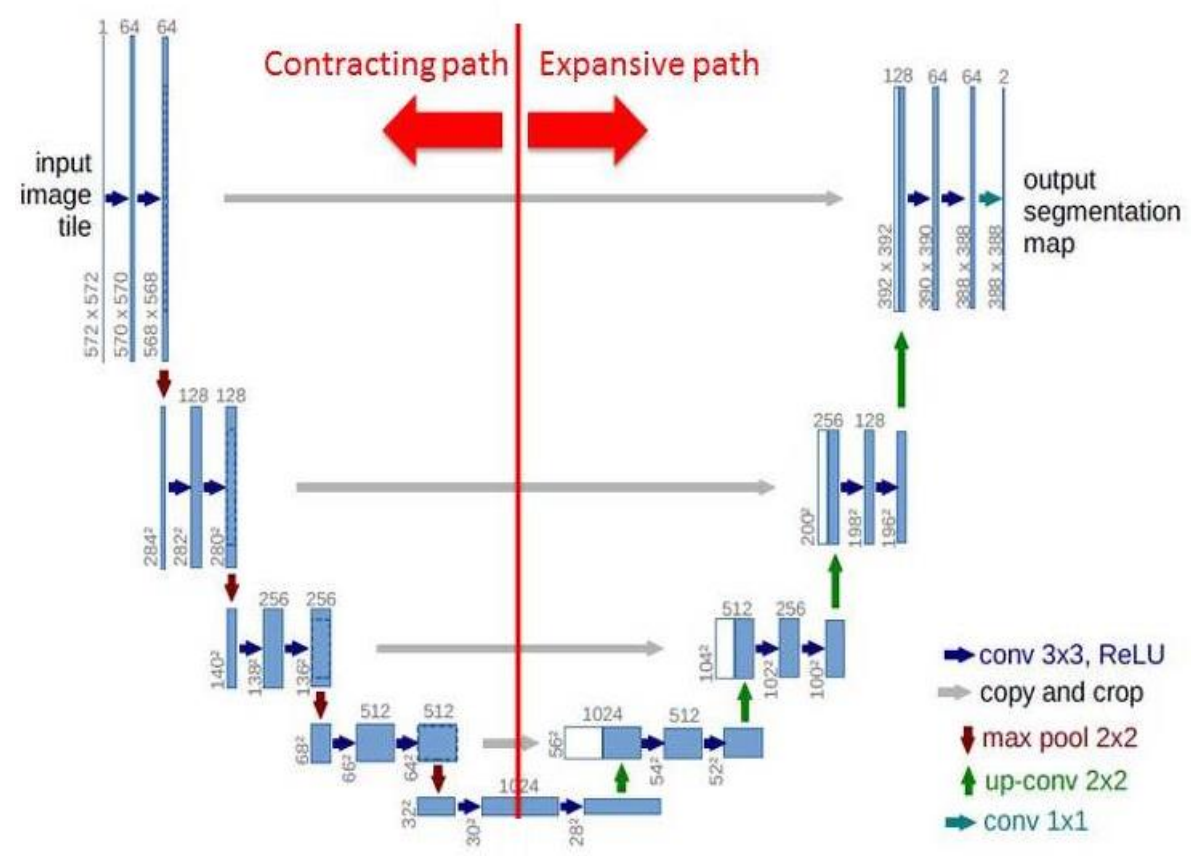

Figure.2 U-Net Architecture

\subsection{Pre-Processing}

CT image is firstly resized to $128 \times 128$ before fitting to the network because all CT image in MICCAI and 3D IRCAD datasets are 512×512, but training such images on personal computer may took very long time to complete. Also any empty images were discarded from both original image and its mask because they would not assistance in training process. Finally, we adding Gaussian noise to CT images which give more variability to it. 


\subsection{Bata-Unet Architecture}

BATA-Unet architecture is based on the U-net architecture described above withe some different which is that each convolutions layer in our proposed network is followed by Batch Normalization layer. Figure. 3 shown our architecture and it consists of contracting path, the middle and expansive path. The contracting path is like in the typical architecture of U-Net network with adding of dropout layer after 2 convolutions and one max-pooling layer. It consists of $3 \times 3$ convolutions repeated two times, each one followed by a rectified linear unit (ReLU) activation, which is following by a $2 \times 2$ max-pooling layer and dropout layer. The purpose of the dropout layer is to avoiding overfitting problem.

After the contracting path there are middle layer consist of convolutions layers The expansive path consists of a transpose convolutional layer or as it known as De- convolution layers and concatenation with the corresponding cropped feature map from the contracting path, followed by $3 \times 3$ convolution repeated two times. This block of De-convolution layers, concatenation and two $3 \times 3$ convolutions layers repeated four times. At the final layer $1 \times 1$ convolution used to map each component feature vector to the desired number of classes.

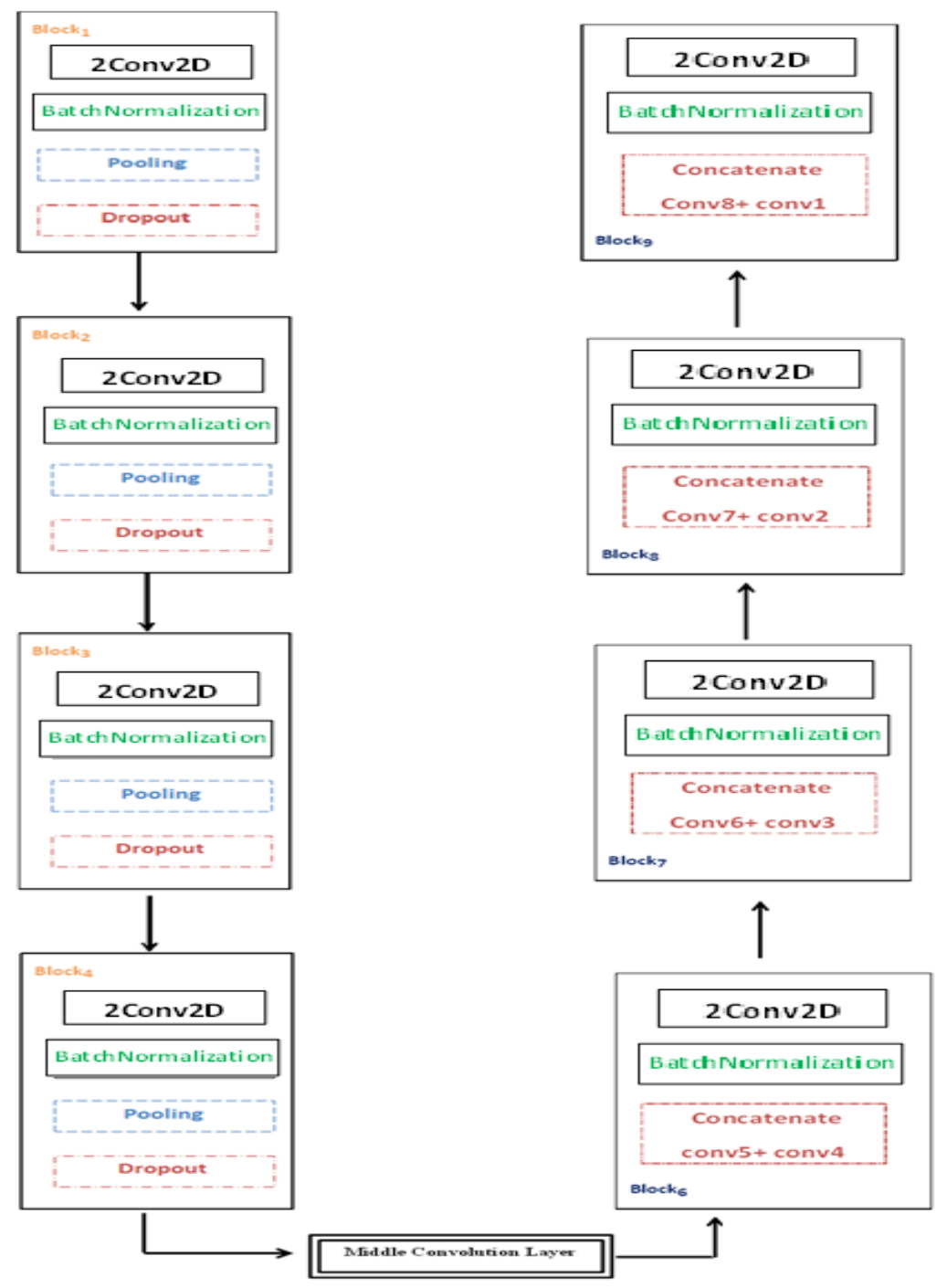

Figure.3 Architecture of Proposed Model 
Signal \& Image Processing: An International Journal (SIPIJ) Vol.11, No.5, October 2020

\subsection{Post-Processing}

To enhance the overall segmentation results we did a post processing step which remove small area in the final segmentation mask by followed two steps. First we calculate the largest connected area in the final segmentation mask to set it as the threshold volume by setting the background pixel intensity to zero and each area that is not zero within the mask is labeled. Then, all the areas less than the threshold are removed.

\section{RESULTS AND DISCUSSION}

The MICCAI and 3DIRCAD datasets was used in the experimental of this research work. the experiments were configured with processor Intel Core(TM) i7-6500U ,8.00 GB memory. the proposed BATA-Unet model consist of 19 convolution layers, 18 BatchNormalization layer and 4 maxpooling layers, 4 concatenate layers, 4 dropout layer and 4 Transposed convolution layer (Deconvolution layer) as well as a softmax layer. For training the network we employed Adam optimizer with learning rate 1e-3. For MICCAI dataset the network was trained for 25 epochs with a batch size of 41 as shown in the accuracy and loss curves in Figure. 4 and for 3DIRCAD dataset the network was trained for 250 epochs with a batch size of 41 as shown in the accuracy and loss curves in Figure.5.
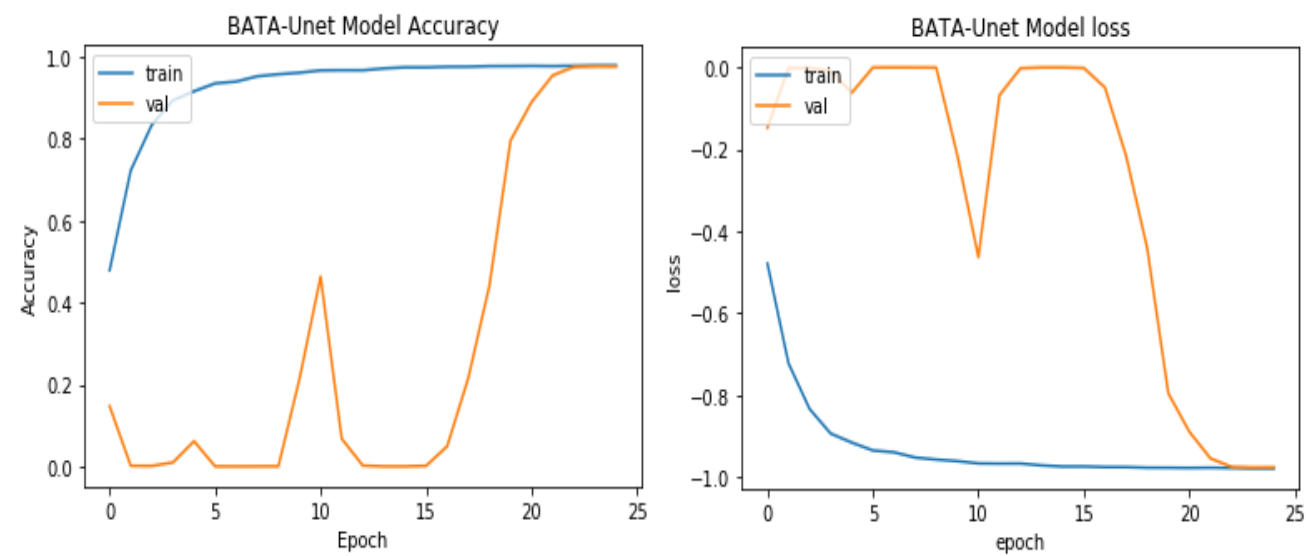

Figure.4 The Training, validation loss and accuracy curves for MICCAI dataset.
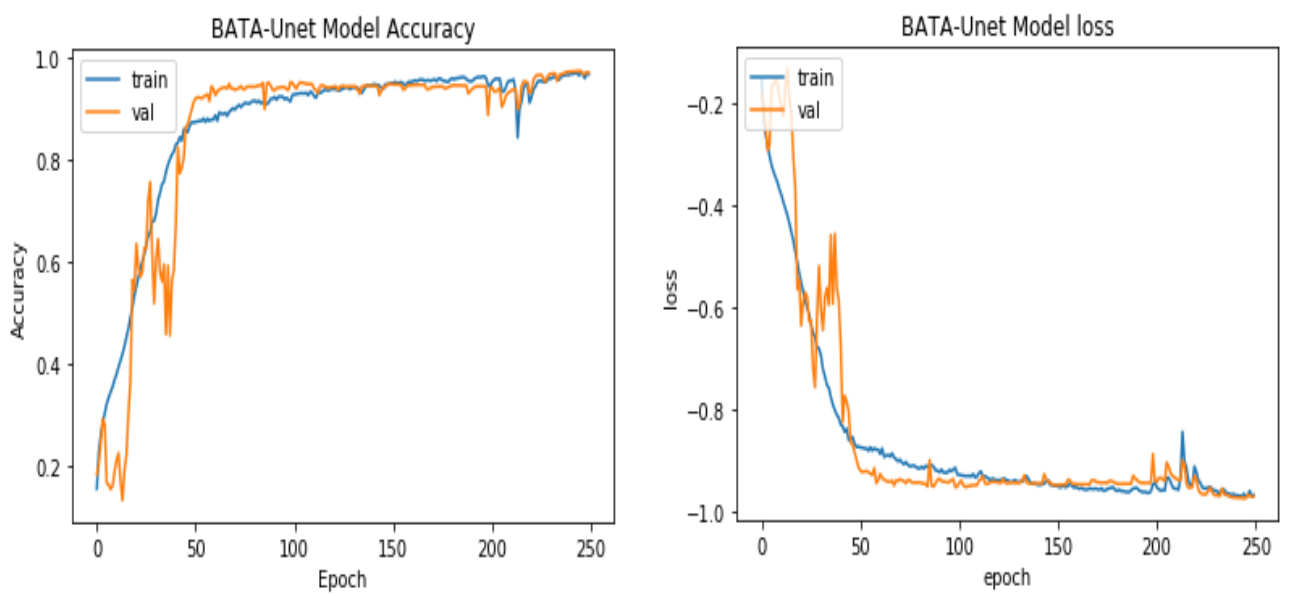

Figure.5 The Training, validation loss and accuracy curves for 3DIRCAD dataset. 
Signal \& Image Processing: An International Journal (SIPIJ) Vol.11, No.5, October 2020

The Dice Similarity Coefficient (Dice), Volume Overlap Error (VOE), Relative Volume Difference (RVD), Average Symmetric Surface Distance (ASD) And Maximum Surface Distance (MSD) were utilized to evaluate the proposed BATA-Unet model and the results are compared with some of related works include Cascaded U-Net [12], EDCNN [19], SBBS-CNN [22], H-DenseUNet [23] and mU-Net [20] as shown in Table 1. It obviously from table 1 that our model is outperformed the other state-of-the-art model for Dice evaluation metrics which it's equals to $97.88 \%$ when tested using MICCAI database and its equals to $96.71 \%$ when tested using 3DIRCAD database. Also for VOE evaluation metrics which it's equals to $4.50 \%$ using MICCAI and $11.50 \%$ using 3DIRCAD. Furthermore, we compare the results with our previous model in [] as shown in Table 2. The results of segmentation it's enhanced when using our new proposed BATA-Unet model, where in our previous work the Dice was equals to $91.05 \%$; it's become $97.88 \%$ for MICCAI. And for 3DIRCAD was equals to $84.68 \%$; it's become $96.71 \%$.

Table1. Comparison between proposed model and some of related works

\begin{tabular}{c|c|c|c|c|c}
\hline Related Work & Dice & VOE & RVD & ASD & MSD \\
\hline Cascaded U-Net [12] & 93.1 & 12.8 & -3.3 & 2.3 & 46.7 \\
\hline Cascaded U-Net + 3D CRF [12] & 94.3 & 10.7 & -1.4 & 1.5 & 24.0 \\
\hline EDCNN [19] & 95.22 & 9.05 & 7.03 & 1.43 & 19.37 \\
\hline SBBS-CNN [22] & 97.31 & 5.24 & 1.97 & 1.77 & 13.03 \\
\hline H-DenseUNet [23] & 94.7 & 10.02 & -0.01 & 4.06 & 9.63 \\
\hline mU-Net [20] & 96.01 & 9.73 & 0.38 & 3.11 & 9.20 \\
\hline Result for MICCAI & $\mathbf{9 7 . 8 8 \%}$ & $\mathbf{4 . 5 0 \%}$ & $\mathbf{0 . 0 4 \%}$ & $\mathbf{0 . 0 5 m m}$ & $\mathbf{0 . 0 8 m m}$ \\
\hline Result for 3D-IRCAD & $\mathbf{9 6 . 7 1 \%}$ & $\mathbf{1 1 . 5 0 \%}$ & $\mathbf{0 . 0 8 \%}$ & $\mathbf{0 . 1 4 m m}$ & $\mathbf{0 . 1 6} \mathbf{m m}$
\end{tabular}

Table2. Comparison between proposed model and our previous works

\begin{tabular}{c|ccccc}
\cline { 2 - 6 } & Dice & VOE & RVD & ASD & MSD \\
\hline $\begin{array}{c}\text { Previous Result for } \\
\text { MICCAI[] }\end{array}$ & $91.05 \%$ & $13.44 \%$ & $0.23 \%$ & $0.29 \mathrm{~mm}$ & $0.36 \mathrm{~mm}$ \\
\hline $\begin{array}{c}\text { BATA-Unet Result } \\
\text { for MICCAI }\end{array}$ & $\mathbf{9 7 . 8 8 \%}$ & $\mathbf{4 . 5 0 \%}$ & $\mathbf{0 . 0 4 \%}$ & $\mathbf{0 . 0 5 m m}$ & $\mathbf{0 . 0 8 m m}$ \\
\hline $\begin{array}{c}\text { Previous Result for } \\
\text { 3D-IRCAD[] }\end{array}$ & $84.68 \%$ & $13.24 \%$ & $0.16 \%$ & $0.32 \mathrm{~mm}$ & $0.33 \mathrm{~mm}$ \\
$\begin{array}{c}\text { BATA-Unet Result } \\
\text { for 3D-IRCAD }\end{array}$ & $\mathbf{9 6 . 7 1 \%}$ & $\mathbf{1 1 . 5 0 \%}$ & $\mathbf{0 . 0 8 \%}$ & $\mathbf{0 . 1 4 m m}$ & $\mathbf{0 . 1 6 m m}$ \\
\hline
\end{tabular}

Figure.6 and Figure.7 are showing some of the Segmentation results of the proposed model for MICCAI and 3DIRCAD respectively. The left column shows original image slices, the middle column shows the ground truth mask and the right column is show the segmentation map obtained using our BATA-Unet model. The overall Segmentation results of the proposed is good but still there are some anomaly case as shown in Figure. 8 and Figure. 8 for MICCAI and 3DIRCAD datasets respectively. 
Signal \& Image Processing: An International Journal (SIPIJ) Vol.11, No.5, October 2020

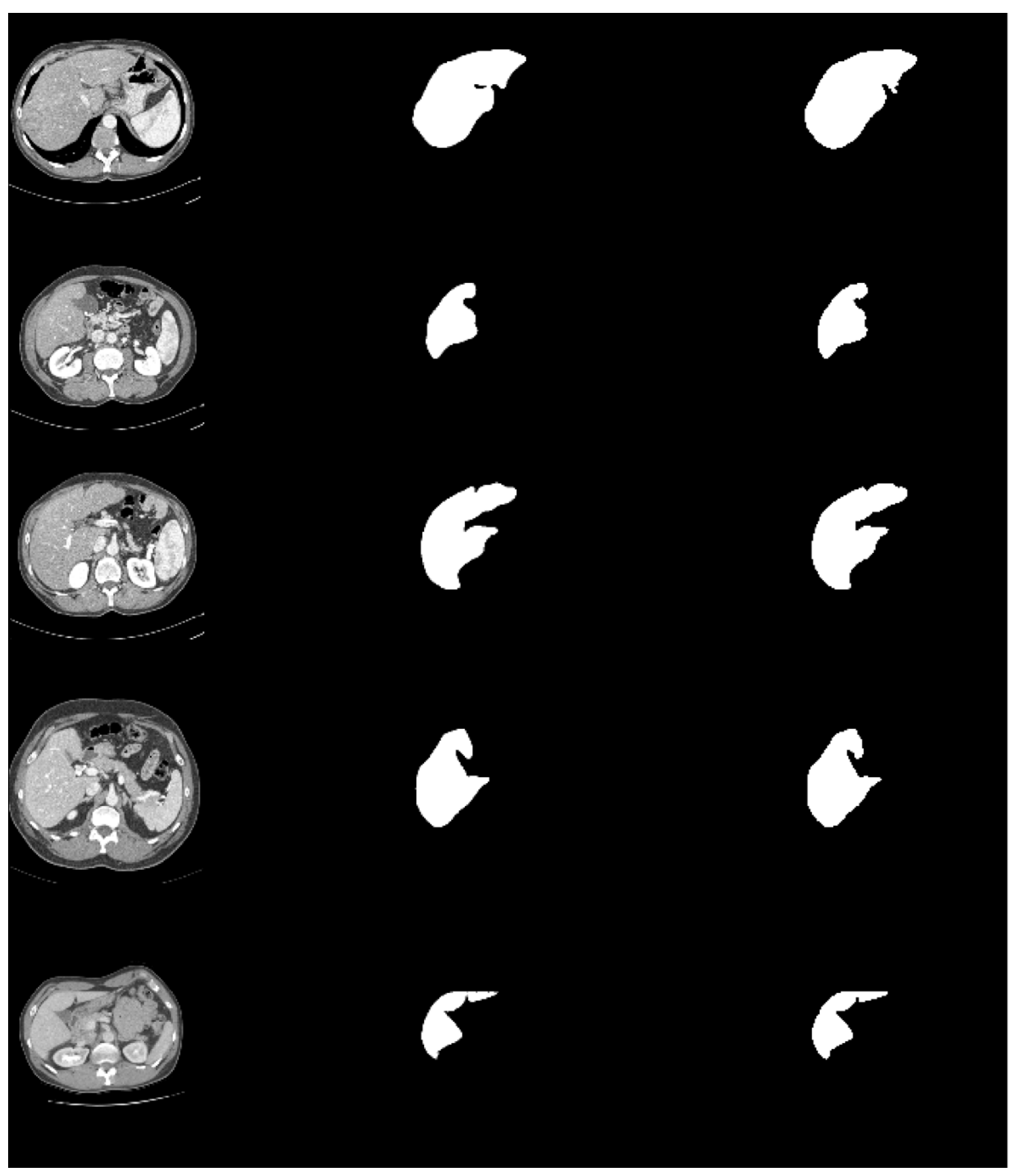

Figure.6 The Segmentation results of the proposed model for MICCAI dataset. 
Signal \& Image Processing: An International Journal (SIPIJ) Vol.11, No.5, October 2020

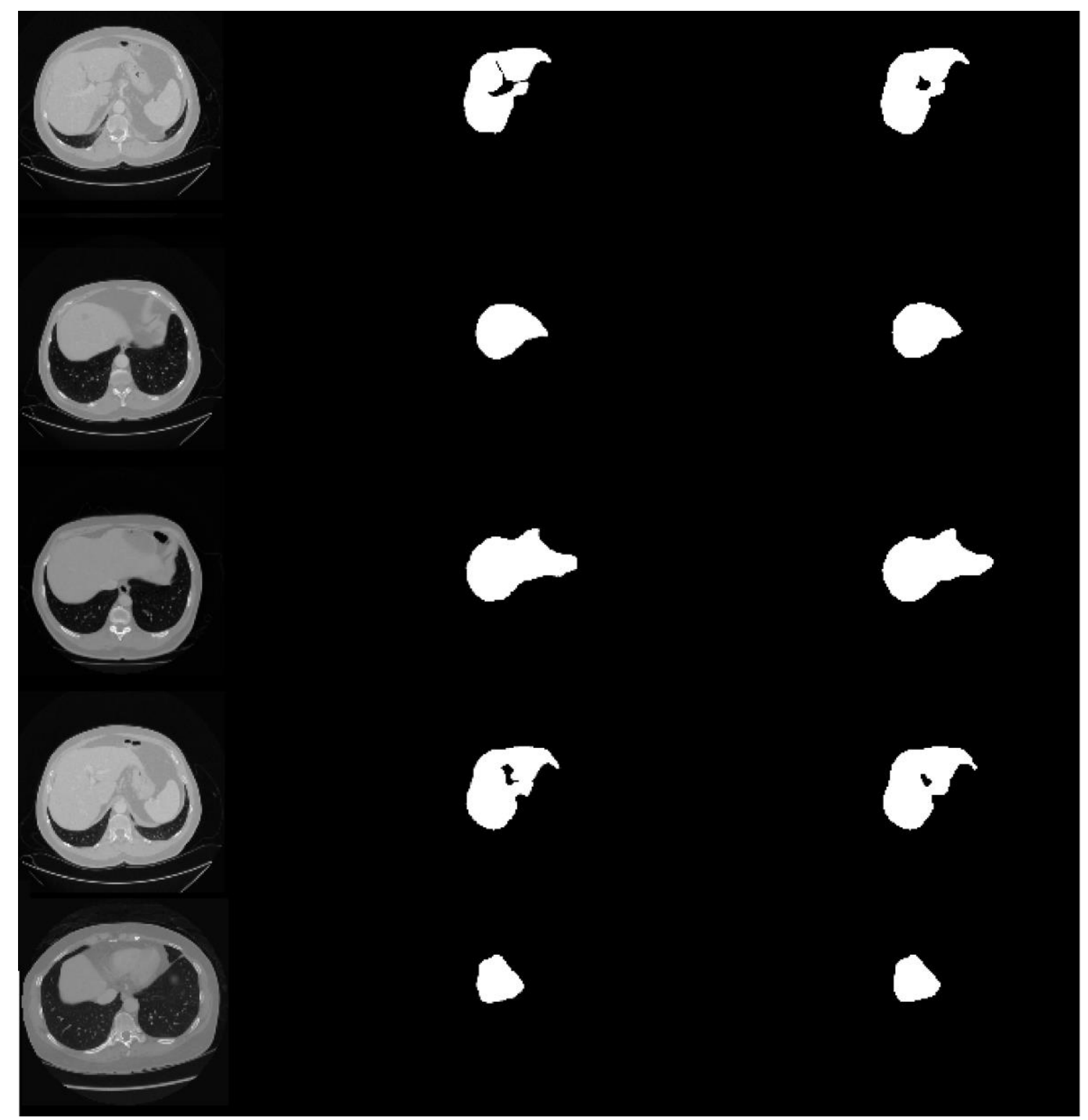

Figure.7 The Segmentation results of the proposed model for 3DIRCAD dataset.
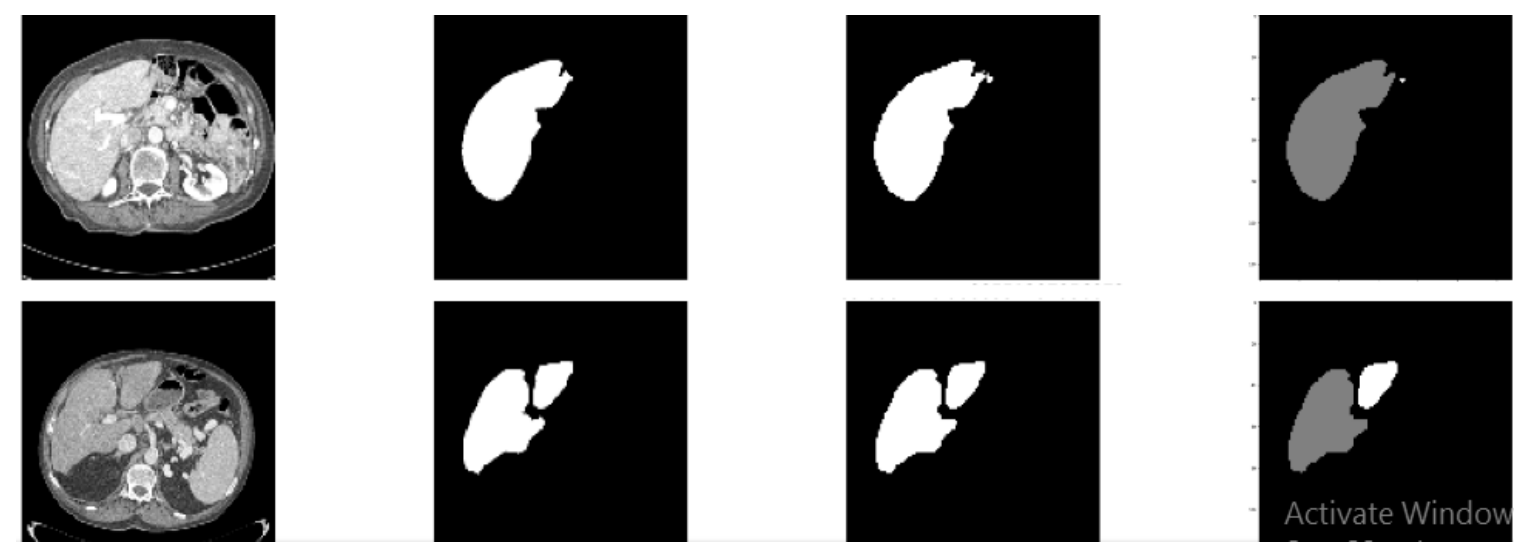

Figure.8 The segmentation result of anomaly case in MICCAI dataset. 
Signal \& Image Processing: An International Journal (SIPIJ) Vol.11, No.5, October 2020
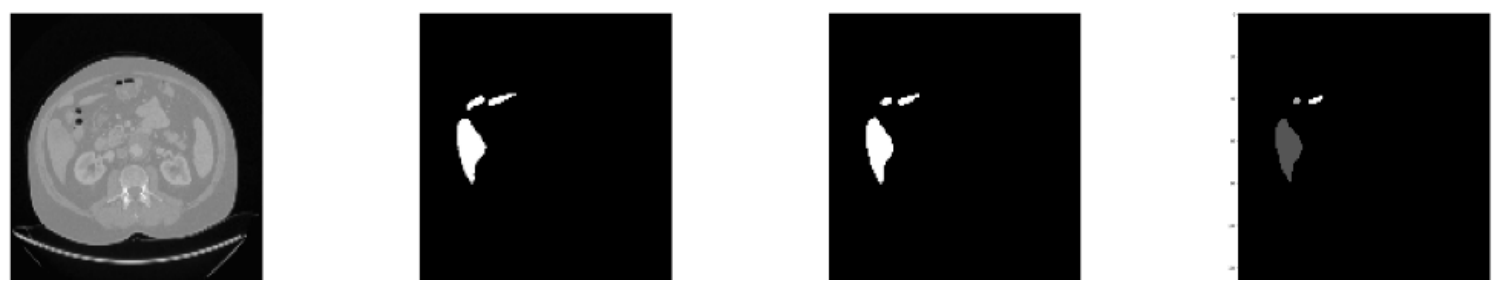

Figure.9 The segmentation result of anomaly case in 3DIRCAD dataset.

\section{CONClusion}

In recent years, the employment of deep learning especially Unet network has been the most promising method for the accurate segmentation of a liver. When we proposed the work in [] the results are good but it's not good enough. So we exploitation the benefit of the Unet architecture and propose a new method for liver segmentation by applying the concept of the Batch Normalization process. According to [24], the Batch Normalization is increase the constancy of the network, which normalizes the inputs to a layer in the network by applying a transformation that subtracting the batch mean and dividing by the batch standard deviation. It's accelerates the training process of the network and it's improved the performance of the model. In this paper we proposed a BATA-Unet model based on the U-net architecture with adding Batch Normalization layer after each convolutions layer as shown in Figure. 3 where the left part of the architecture is the contracting path and the right part the expansive path. The proposed BATA-Unet model was able to achieve highest dice similarity coefficient than the previous work where for MICCA dataset Dice $=0.97 \%$ and for 3D-IRCAD dataset $=0.96 \%$. Also we compare it with other state-ofthe-art model and it's outperformed them. In future, we hope to study the anomaly cases and try to specify the reason of that and fixed it which further increase the accuracy of the segmentation.

\section{CONFLICT OF INTEREST}

The authors declare no conflict of interest.

\section{REFERENCES}

[1] J. A. P, “Computer Vision and Medical Image Processing :," pp. 152-159, 2015.

[2] D. Wong et al., "A semi-automated method for liver tumor segmentation based on 2D region growing with knowledge-based constraints,” Midas J., no. January, 2008.

[3] L. Hermoye et al., "Liver Segmentation in Living Liver Transplant Donors: Comparison of Semiautomatic and Manual Methods," Radiology, vol. 234, no. 1, pp. 171-178, 2005.

[4] T. Heimann, I. Wolf, and H.-P. Meinzer, "Active shape models for a fully automated 3D segmentation of the liver--an evaluation on clinical data.," Proc. Med. Image Comput. Comput. Interv., vol. 9, no. Pt 2, pp. 41-8, 2006.

[5] L. Massoptier and S. Casciaro, "A new fully automatic and robust algorithm for fast segmentation of liver tissue and tumors from CT scans," Eur. Radiol., vol. 18, no. 8, pp. 1658-1665, 2008.

[6] F. Chollet, Deep Learning with Python, vol. 10, no. 2. 2011.

[7] P. Lakhani, D. L. Gray, C. R. Pett, P. Nagy, and G. Shih, "Hello World Deep Learning in Medical Imaging," 2018.

[8] G. Litjens et al., “A Survey on Deep Learning in Medical Image Analysis,” no. February, 2017.

[9] M. Mazurowski and M. Buda, "Deep learning in radiology: an overview of the concepts and a survey of the state of the art," no. March, 2018.

[10] P. Hu, F. Wu, J. Peng, P. Liang, and D. Kong, "Automatic 3D liver segmentation based on deep learning and globally optimized surface evolution,” Phys. Med. Biol., vol. 61, no. 24, pp. 8676-8698, 2016. 
Signal \& Image Processing: An International Journal (SIPIJ) Vol.11, No.5, October 2020

[11] F. Lu, F. Wu, P. Hu, Z. Peng, and D. Kong, "Automatic 3D liver location and segmentation via convolutional neural networks and graph cut," 2016.

[12] P. F. Christ et al., "Automatic Liver and Tumor Segmentation of CT and MRI Volumes using Cascaded Fully Convolutional Neural Networks," pp. 1-20, 2017.

[13] and H. G. Avi Ben-Cohen, Idit Diamant, Eyal Klang, Michal Amitai2, "Fully Convolutional Network for Liver Segmentation and Lesions Detection," vol. 1, pp. 77-85, 2016.

[14] M. Chung, J. Lee, J. Lee, and Y.-G. Shin, "Liver Segmentation in Abdominal CT Images via AutoContext Neural Network and Self-Supervised Contour Attention," 2020.

[15] N. Ibtehaz and M. S. Rahman, "MultiResUNet: Rethinking the U-Net architecture for multimodal biomedical image segmentation," Neural Networks, vol. 121, pp. 74-87, 2020.

[16] G. Du, X. Cao, J. Liang, X. Chen, and Y. Zhan, "Medical image segmentation based on U-Net: A review," J. Imaging Sci. Technol., vol. 64, no. 2, pp. 1-12, 2020.

[17] Z. Zhou, M. M. Rahman Siddiquee, N. Tajbakhsh, and J. Liang, "Unet++: A nested u-net architecture for medical image segmentation," Lect. Notes Comput. Sci. (including Subser. Lect. Notes Artif. Intell. Lect. Notes Bioinformatics), vol. 11045 LNCS, pp. 3-11, 2018.

[18] S. Shrivastava, N. Singh, U. Mishra, A. Chandra, and S. Verma, "Comparative Study of Deep Learning Models for Segmentation of Corpus Callosum," Proc. 4th Int. Conf. Comput. Methodol. Commun. ICCMC 2020, no. Iccmc, pp. 418-423, 2020.

[19] Ü. Budak, Y. Guo, E. Tanyildizi, and A. Şengür, "Cascaded deep convolutional encoder-decoder neural networks for efficient liver tumor segmentation," Med. Hypotheses, vol. 134, no. September 2020.

[20] H. Seo, C. Huang, M. Bassenne, R. Xiao, and L. Xing, "Modified U-Net (mU-Net) with Incorporation of Object-Dependent High Level Features for Improved Liver and Liver-Tumor Segmentation in CT Images," IEEE Trans. Med. Imaging, vol. 39, no. 5, pp. 1316-1325, 2020.

[21] O. Ronneberger, P. Fischer, and T. Brox, "U-Net: Convolutional Networks for Biomedical Image Segmentation," Miccai, pp. 234-241, 2015.

[22] W. Qin et al., "Superpixel-based and boundary-sensitive convolutional neural network for automated liver segmentation,” Phys. Med. Biol., vol. 63, no. 9, 2018.

[23] X. Li, H. Chen, X. Qi, Q. Dou, C. W. Fu, and P. A. Heng, "H-DenseUNet: Hybrid Densely Connected UNet for Liver and Tumor Segmentation from CT Volumes," IEEE Trans. Med. Imaging, vol. 37, no. 12, pp. 2663-2674, 2018.

[24] S. Ioffe and C. Szegedy, "Batch normalization: Accelerating deep network training by reducing internal covariate shift,” 32nd Int. Conf. Mach. Learn. ICML 2015, vol. 1, pp. 448-456, 2015. 